\title{
La Inclusión de la Perspectiva de la Sociedad Civil Popular en VIH/SIDA: sistematización de experiencias como enfermera en Brasil y Canadá'
}

\section{Inclusion of Popular Civil Society Perspective in HIV/AIDS: sistematization of experiences as a nurse in Brazil and}

\section{Canada}

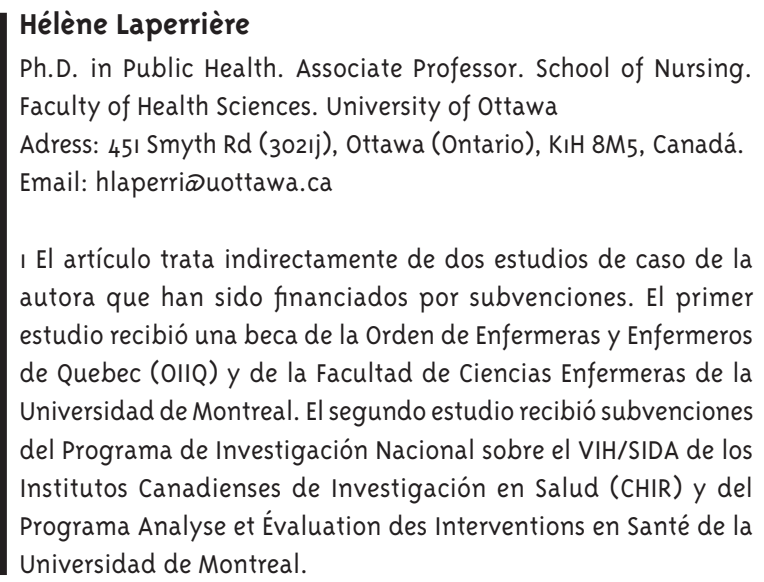

Ph.D. in Public Health. Associate Professor. School of Nursing. Faculty of Health Sciences. University of Ottawa

Adress: 45I Smyth Rd (3021j), Ottawa (Ontario), KıH 8M5, Canadá.

Email: hlaperriळuottawa.ca

I El artículo trata indirectamente de dos estudios de caso de la autora que han sido financiados por subvenciones. El primer estudio recibió una beca de la Orden de Enfermeras y Enfermeros de Quebec (OIIQ) y de la Facultad de Ciencias Enfermeras de la Universidad de Montreal. El segundo estudio recibió subvenciones del Programa de Investigación Nacional sobre el VIH/SIDA de los Institutos Canadienses de Investigación en Salud (CHIR) y del Programa Analyse et Évaluation des Interventions en Santé de la Universidad de Montreal.

\section{Resumen}

La enfermera en Salud Pública tiene como misión movilizar las organizaciones de base de su país para implantar los programas de salud. Este trabajo implica un acercamiento que le permita captar el detalle de la realidad concreta, pero que sólo una cierta distancia crítica le permitirá situarlo en un contexto de significación global. Una sistematización de experiencias presenta la investigación evaluable comunitaria como una oportunidad metodológica para las ciencias de la salud en general y de enfermería. La perspectiva es especialmente pertinente para las prácticas profesionales de salud, específicamente en contextos imprevisibles y de conflictos de verticalidad sociopolítica entre los diversos actores implicados en un programa de salud pública. La inclusión en barrios periféricos y el trabajo con grupos comunitarios favorece la inclusión desde el punto de vista de la sociedad civil popular dentro de la evaluación de las acciones preventivas del Sida y de otros programas de salud pública.

Palabras-claves: Salud pública y colectiva; Sociedad civil popular; Movimientos sociales; Investigación evaluable comunitaria; enfermera; Verticalidad sociopolítica; Imprevisión; Prevención del Sida. 


\section{Abstract}

Nurses in Public Health have the mission to mobilize the community-based organizations for the implementation of national health programmes. The work engages a proximity that will allow them to seize the details of a concrete reality, but which only a certain critical distance will lead to place them in a context of global meaning. A systematization of experiences presents the community evaluative research as a methodological opportunity for health and nursing sciences. The perspective is particularly relevant for health professional practices, especially in contexts of unpredictability and of sociopolitical conflicts of verticality among multiple actors involved in a public health program. The field insertion in marginalized districts and the work with community groups favor the inclusion of the popular civil society perspective in evaluations of AIDS preventive actions and other public health programmes.

Keywords: Public and Community Health; Popular Civil Society; Social Movements; Community Evaluative Research; Nursing; Sociopolitical Verticality; Unpredictability; Aids Prevention.

\section{Introducción}

La Organización Mundial de la Salud (WHO, 1986) favorece el compromiso de las organizaciones de base de la sociedad civil en la promoción de la salud. La Carta de Bangkok (WHO, 2006) solicita hoy la cooperación entre los sectores de la sociedad civil (sector voluntario), las agencias gubernamentales de salud (sector público) y las empresas comerciales (sector privado) para incorporar una perspectiva de desarrollo económico en las prácticas de promoción de la salud. Este movimiento en la salud pública sigue la prescripción de respuestas locales y multisectoriales para la lucha contra el Sida (UNAIDS, 2005). La OMS, el Banco Mundial y las Naciones Unidas para el desarrollo solicitan en conjunto a todos los gobiernos de los países llamados "en desarrollo", "en transición"o"desarrollados" a incluír a la sociedad civil en la implantación de los programas de salud pública (UNAIDS/WB/UNDP, 2006). Las ideas generales de participación igualitaria y compromiso confrontan y complican las discusiones sobre la transparencia y la imputación de los programas de lucha contra el SIDA (WHO, 2006; UNAIDS, 2005; UNAIDS/WB/UNDP, 2006; UNDP, 2004). Las organizaciones de la sociedad civil enseñan y preparan para la participación a las organizaciones de base en las decisiones locales, regionales, nacionales e internacionales (ICASO, 2007).

Tampoco hay soluciones sencillas para las intenciones de intervención que conllevan presupuestos ingenuos como que el cambio propuesto sea necesariamente mejor que las soluciones vigentes en las situaciones que confrontan las organizaciones de base de las diversas poblaciones. La crítica al "desarrollismo" ya ha sido ampliamente comentada tanto por autores Latinoamericanos como Norteamericanos en las ciencias sociales y de la salud (Horowitz, 1974; Zúñiga, 1976; Stavenhagen, 1981, Stavenhagen, 1992; Diamond, 1999, Casanova, 2002; Farmer, 2001; Farmer, 2003). Demo (2000) denuncia la obsesión innovadora del conocimiento científico que lo lleva a atribuirse el derecho de imponer cambios específicos sin considerar el conjunto social en el que se inserta y, por otra parte, las desigual- 
dades entre los países ricos y los pobres así como entre las élites nacionales y los sectores populares, que se reflejan en la accesibilidad diferente a los conocimientos y a las posibilidades de producirlos y de difundirlos. El plantea también, el creciente problema de la "marginalización digital" de grandes sectores (Demo, 2007).

El problema que enfrentamos es el de las condiciones de la posibilidad de la transferencia de experiencias particulares de la sociedad civil de carácter "popular" para la generalización de conocimientos empíricos legítimos de nuestros programas de salud pública. Victor Vincent Valla (2006, p. 269) distingue la sociedad civil popular de la sociedad civil representada por entidades empresariales o estatales. La sociedad civil popular corresponde más adecuadamente a los grupos comunitarios y a los movimientos. Hay una confusión frecuente en los medios académicos entre proximidad y subjetividad, por una parte, y distancia y objetividad, por otra. Suponer que acercarse a la experiencia del otro no produce nada más que distorsión "subjetiva” y contaminación de los datos y que mantenerse a distancia es una garantía de "objetividad" y de pureza de los datos, es una confusión semántica que tiene más del uso descuidado del lenguaje que de un análisis riguroso (Zúñiga, 2008).

Para conocer, como para actuar, hay que acercarse y tomar contacto (Vasconcelos, 2008). Que ésta aproximación pueda ser fuente de fusión de intereses no es más cierto que el que la distancia pueda ser fuente de indiferencia y de incomprensión. La investigación en salud pública confronta los desafíos metodológicos de estudiar los contextos y las sociedades complejas incluidas en ellos que exigen intervenciones mucho más específicas que hacen mucho más difícil la comparación y la utilización de grupos de control (Petticrew y col., 2005; Alvarado y col. 2009). Otros autores han subrayado la perspectiva del "pueblo laico" (Entwistle y col., 1998) y del potencial que tiene para contribuir en las investigaciones: ellos conocen en su cotidianidad las situaciones que viven, principalmente en contextos de vulnerabilidad social y de pobreza (Vasconcelos, 2008; Silva y Bessa, 2008; Titterton y Smart, 2008).
Las situaciones complejas y particulares necesitan ser abordadas a través de prácticas en enfermería que estén más finamente sintonizadas con la realidad local (Salas, 2005).

El texto está organizado en dos pasos. El primero es el análisis de los dos casos que fueron el terreno de los intercambios entre participantes y la investigadora (en su rol de enfermera de salud pública). Este paso identifica y analiza los conceptos que emergieron como relevantes para entender mejor los desafíos de la evaluación a la que puede contribuir la sociedad civil popular. El segundo es una síntesis reflexiva sobre las perspectivas de incorporación productiva de la enfermera en salud pública a la función de la sociedad civil popular (grupos comunitarios) al enriquecimiento de una evaluación unida a acciones sociales de prevención del Sida.

Este artículo pretende mostrar primero la riqueza que ha aportado la perspectiva de la sociedad civil popular en la evaluación de las acciones sociales de lucha contra el Sida: El análisis ha sido estructurado por medio de la sistematizacion de experiencias de dos investigaciones participativas realizadas por la autora (voluntaria, enfermera, investigadora) con trabajadoras del sexo en pueblos del Amazonas (Brasil) y con grupos de lucha contra el Sida en Canadá. La tesis subyacente es que la acción social comunitaria de la sociedad civil popular enriquece la acción gubernamental de prevención del Sida, cuando ella desarrolla y mantiene la conciencia de la validez y de la fertilidad de la convergencia de ambas perspectivas.

El artículo también subraya que los profesionales de primera línea en salud pública tienen una posición privilegiada para incluír la perspectiva de la acción social comunitaria de la sociedad civil popular en el conjunto del conocimiento científíco en salud publica. Especialmente, cuando las acciones se desarrollan en contextos caracterizados por situaciones de peligro, imprevisibles y en organizaciones sociopolíticas verticales, que frecuentemente limitan su validez cientifica al descuidar variables locales que exigen mayor fineza en la definición de conocimientos que pretendan ser universales. 
Material y Método: la

sistematización de experiencias

de dos casos de evaluación

\section{comunitaria}

La estrategia metodológica de la sistematización permite examinar una realidad conflictiva y contradictoria que está presente en la acción de un proyecto colectivo para percibir mejor el futuro de la situación que se desea cambiar (Zúñiga, 2009; Alboan, 2006a; Alboan, 2006b). La sistematización es la interpretación crítica de una o más experiencias que, a partir de su organización y de su reconstrucción, descubre la lógica del proceso vivido colectivamente, los factores que han estado en juego en el proceso y cómo ellos se han influenciado mutuamente (Eizaguirre, Urrutia y Askunze, 2004; Jara, 2006). El método propone una ética social de compromiso personal y colectivo, que favorece la circulación pública de las experiencias de medios informales, como es el caso de la circulación de aprendizaje de muchos grupos comunitarios y movimentos de la sociedad civil popular (Zúñiga, 2009).

En este estudio, el término "investigación" se utiliza por tener el componente de práctica reflexiva que permite avanzar hacia un análisis más formalizado y más profundo (investigar) de las que fueron experiencias de "evaluación" (sistematizar) que se realizaron en forma "colectiva" con la sociedad civil popular (reflexionar juntos) durante sus acciones sociales de prevención del Sida (actuar). Las dos investigaciones, en las que la autora fue también la investigadora principal, fueron abordadas desde una perspectiva metodológica etnográfica, con una introducción personal intensa: en Brasil, 5 meses de organización formal en actividades de trabajo grupal de interpretación participativa, que siguieron una inclusión inicial de 6 años ininterrumpidos como habitante en la comunidad y como enfermera en salud pública local y en Canadá, con 2 años que incluyeron más de 400 horas de actividad como voluntaria y 127 visitas en el terreno a los diferentes grupos. La opción por este enfoque tiene una función importante al permitir acercarse con mayor detalle a la realidad concreta de las situaciones en las que se realiza un proyecto con la sociedad civil popular (Vasconcelos, 2008). Gracias a ella, se puede observar con más detalles la relación que hay entre los resultados obtenidos y el grado de incorporación de todos los participantes en una evaluación que privilegia una participación que permita una relación de participación igualitaria. La investigación se revela como una forma de enriquecimiento colectivo, que puede hacer de un proyecto una realidad compartida (Demo, 2008; Brandão, 2006).

Los resultados de esta sistematización de experiencias están basados en tres dimensiones interrelacionadas: (a) descripciones micro-contextuales, (b) realización de intercambios dialogantes en profundidad, y (c) la emergencia de las subjetividades de todos los interlocutores, hacer explícita la posición de la investigadora y discutir con franqueza las posibilidades reales de transformación (33). "Todo quehacer profesional requiere desarrollar procesos de aprendizaje basados en sus prácticas de acción" (Asún, 2009, p.5) con su aproximación a la experiencia cotidiana.

\section{El Caso de una Comunidad Evaluable Emergente: lo imprevisible y los controles verticales en la región del Amazonas (Brasil)}

Una evaluación por los pares de un proyecto de prevención de enfermedades transmitidas sexualmente centrado en la prevención del Sida en región amazónica brasileña (Laperrière, 2008a, 2008b) subraya esta importancia de la convergencia de objetividad y subjetividad como potencial de recuperación de la historia colectiva. Dado que el proyecto ya había sido evaluado anteriormente por encuestas epidemiológicas y cuestionarios evolutivos sobre las conductas sexuales (Benzaken y col., 2002), el proceso de evaluación cualitativo participativo subrayó los impactos secundarios imprevistos del proyecto (Scriven, 2004) y la construcción de significaciones colectivas unidas a la implantación inicial hecha por los pares. 
La proximidad con el medio local favoreció la inclusión en el movimiento social del contexto sociopolítico, primero en la acción comunitaria (1994 a 2000) y luego en la evaluación del proyecto (2004). Durante un trabajo de cinco meses, la observación participante, las entrevistas individuales y colectivas de una variedad de actores locales (implicando intensamente a 35 personas) por medio de útiles de autoevaluación (Jalbert y col., 1997) permitieron hacer emerger otras dimensiones del impacto global del proyecto en el medio sociopolítico en el que se desarrolló.

El punto de partida fue la identificación de la variedad de actores para incorporarlos al proceso participativo de la evaluación: los usuarios, los agentes multiplicadores y la estructura social de actividades en los puntos de prostitución, la red sociosanitaria, los aparatos jurídicos y políticos como los administradores públicos, policías y militares, los aparatos culturales, los políticos y el personal de investigación, y la variedad de organizaciones ilícitas que rondan la prostitución y sus lazos con los poderes públicos y fácticos.

Esta enumeración de actores que son parte integral de un proyecto de prevención muestra la futilidad y el peligro de una creencia ingenua en la autonomía social de una investigación que ignoraría los lazos entre las líneas de actividades sociales, económicas y políticas, sus controles y su legitimidad. La estrategia para abordar el terreno fue la de un plan operacional que estuvo siempre en interacción con el conocimiento progresivo de los grados de peligro de los contextos sociocultural, sociopolítico y geográfico en el que estaba incluido el proyecto (presencia de informadores de poderes políticos y delictivos). Expresando puntos de vista y opiniones personales, los participantes (incluyendo a la investigadora) podían efectuar un proceso de objetivación (Laperrière, 2008a), al menos de su discurso público, siempre con cuidado por las consecuencias de herir susceptibilidades políticas. La participación de los actores locales en el proceso evaluable permitió identificar importantes variantees contextuales contribuyentes con las acciones preventivas, otras de aquellas elaboradas por el programa del proyecto de prevención y así mejor arraigar su contribución en condiciones concretas, siempre mucho más claras para los participantes que para la planificación a la distancia.

La experiencia brasileña ha sido analizada bajo el doble prisma de la irrupción de imprevistos sociales y de la verticalidad de las relaciones entre los actores. Los proyectos de prevención permiten insertarse en la realidad estructural de las culturas organizacionales locales, que incluyen aspectos que el investigador no puede marginalizar, como la historicidad de estas organizaciones que define la visión de los participantes. El concepto de verticalidad busca subrayar que, fuera de los canales jerárquicos explícitos e implícitos, están los poderes reconocidos informalmente, que en la región del estudio incluyen los narcotraficantes, los propietarios de los bares en los que se establece la prostitución, las bandas callejeras y los políticos locales, con los que el proyecto de investigación no puede negociar como lo hace con los pares "visibles y reconocidos" del sistema oficial de salud.

Los actores locales son mucho más lúcidos con respecto a las posibilidades y a los ajustes necesarios para realizar los proyectos que han sido formulados en los niveles superiores del aparato sanitario, asegurándole las posibilidades realistas de participación en sus estructuras organizacionales y culturales locales. El sociólogo Pedro Demo (2001, p. 125) fundamenta bien la ncesidad de sobrepasar la noción de participación "ideológica y teórica" de las élites sociales, ya que "una participación sin autosustentación es una farsa".

Esta experiencia fue rica en aprendizaje sobre la lógica evaluable utilizada y su potencial para ser contextualizada en un medio dado. Ella ha llevado a la investigadora a insistir sobre la multiplicidad de los actores implicados en la cotidianidad del proyecto, actores situados en relaciones desiguales de control y de influencia, en una "verticalidad" relativa de poderes acentuada por el "contexto de lo imprevisto”, muy lejano a la lógica dominante de un diseño de investigación que presuponía el control de la situación que era el objeto de la evaluación. 
El Caso de las Desigualdades entre lo Local y lo Nacional en la Evaluacion Participativa de la Lucha Comunitaria Contra el Sida en Quebec (Canadá): formalización impuesta sobre la racionalidad implícita del vivir cotidiano

El segundo caso que nos sirve de material empírico de reflexión y de teorización es el de las prácticas evaluables formales de organismos comunitarios de lucha contra el SIDA en Quebec (Zúñiga y Luly, 2005). La investigación evaluable participativa y colectiva se extendió durante dos años en la forma de un compromiso voluntario de la investigadora con personas enfermas de Sida (2005-2007).

En el año 2004 había sido invitada a un comité de trabajo, formado por la Coalición, para preparar un guía de formación, que se llamaría "Saber hacer y saber decirlo" (Zúñiga y Luly, 2005). Las primeras formulaciones de interrogantes para la tesis emergen de las discusiones con los participantes de este comité. Los intercambios llevan también a visitar diversos medios comunitarios en la Provincia de Quebec. En 2005 se presentó en la Asamblea General de la Coalición la intención de realizar como tesis de doctorado una investigación que se orientaría según las preocupaciones expresadas por los grupos comunitarios sobre el problema específico que representaba para ellos situar la evaluación comunitaria en el contexto y el encuadre impuesto por los organismos estatales que financian los proyectos. En el mismo año comenzó un período de inclusión en dos grupos comunitarios entre los que aceptaron la participación de la investigadora en sus actividades cotidianas, con visitas de trabajo una o más veces por semana.

Además de estos primeros dos grupos, otros tres se unieron a ellos, lo que permitió aumentar la diversidad de medios de práctica. Los cinco grupos participaron en entrevistas colectivas. Ellos son identificados como pertenecientes a (1) Medio urbano en región, (2) Medio metropolitano, (3) Medio semiurbano en región, (4) Medio urbano/rural y (5) Medio metropolitano trabajando con clientelas afec- tadas también por toxicomanías y extrema pobreza. En total son 47 personas las que estuvieron directamente implicadas en 13 "Grupos de apreciación compartida”. Tanto la recolección como el análisis de los datos cualitativos se realizaron en una relación igualitaria entre los participantes y la investigadora durante un mismo período. La utilización de metáforas es la primera estrategia de reducción rápida de los datos. En la práctica, se planteó una frase o un título evocador encabezando los párrafos de un primer informe en dos o tres páginas en las que agrupé las informaciones y los juicios emitidos en la reunión precedente. Los temas pudieron ser luego discutidos en el grupo y modificados colectivamente, para que respondieran al recuerdo y al énfasis de los participantes.

La situación confrontada era la de cómo comprobar el trabajo realizado a la fuente de financiación gubernamental. Los programas gubernamentales subvencionados funcionan con formularios rígidos, genéricos, centrados en la producción de estadísticas de productividad del organismo como organización formal - la voz de la Dirección General del organismo comprobando la utilización de fondos en función de los resultados alcanzados.

Las experiencias comunitarias no pueden ignorar ésta tensión. Los grupos comunitarios siempre actúan con plena conciencia de su doble arraigo: en la comunidad con la que se identifican y en las relaciones contractuales que establecen en el proceso de obtener sus recursos financieros. La tentación más fácil es la estrategia esquizoide: un discurso interno de complicidad solidaria y otro, público, destinado a responder a las expectativas de sus fuentes de financiación. El marco restrictivo del informe esperado se limita a una recolección de cifras de producción de resultados predeterminados (el objetivo concebido como un contrato), y los interlocutores gubernamentales dejan muy claro que ésta es toda la evaluación que esperan. El efecto nefasto de esta realidad y de su percepción es hacer superflua la palabra escrita para la mayoría de los participantes.

Los grupos comunitarios tienen muy claro que confrontan una triple tarea: desarrollar una voz autónoma (la misión del organismo como integración de los programas subvencionados), alimentar internamente la acción y enriquecer el discurso de 
justificación. El desafío es hacer de ellas tareas que sean vistas por el conjunto de sus miembros como modalidades de búsqueda y de expresión de la razón de ser misma de los grupos. Ello implica una tarea antes no confrontada en buscar una palabra que corresponda a las tareas reales, que capte las actividades en su riqueza concreta antes de decidir qué aspectos conservar en el discurso oficial.

El proyecto de desarrollar útiles de evaluación ha trabajado durante varios años para enfrentar esta tarea. Dos grupos de trabajo sucesivos, reagrupando representantes de una docena de organismos cada uno, han trabajado desde 1995 hasta hoy en desarrollar una perspectiva compatible con la doble tarea de ofrecer los servicios programados y de situarlos en el contexto de una globalidad social y comunitaria (Jalbert y col., 1997). Ambos grupos han estado siempre en contacto agridulce con un grupo "consejo", paritario, entre representantes de la Coalición y representantes de las fuentes de financiación federal y provincial, muchas veces marcado por discrepancias de perspectivas que están cuidadosamente en un segundo plano para no impedir un trabajo colectivo. Si bien se han evitado las fricciones, siempre ha estado claro que cada acción de evaluación autónoma debía realizarse en el marco de los criterios de evaluación y de las exigencias de productividad de los programas federales y provinciales que la financian.

El trabajo de construcción de útiles de evaluación es un reflejo de una relación de diálogo permanente con muchos de los cuarenta grupos de la Coalición (Zúñiga y Luly, 2005). Los miembros de los grupos de trabajo han compartido sus reflexiones y también las demandas que han recibido de orientación y de apoyo en éste "saber decir". Se han organizado reuniones de información y jornadas de trabajo con directivos, empleados, voluntarios, usuarios e intermediarios para analizar tanto las necesidades como el valor práctico de los útiles de evaluación propuestos. Los avances más significativos fueron el comprender cómo una evaluación es ante todo una toma de conciencia de las posiciones sostenidas y de las acciones realizadas hacia la realización de un proyecto de cambio y como esta evaluación es siempre una tensión entre los actores directamente implicados en la acción transformadora y los actores que los encuadran, dirigen y controlan - actores igualmente legítimos y necesarios, pero actores con puntos de vista que necesitan una comprensión interna de lo que encuadran. La evaluación es, así, necesariamente interna y externa, no sólo por la posición de los diversos actores, sino también y más profundamente porque la intención transformadora debe escuchar la realidad que busca transformar: todo actor autónomo es responsable de los fines, de los medios que elige y de la constatación del resultado de sus esfuerzos.

\section{Discusión y Comentarios}

La distancia entre quien evalúa y quien ejecuta refleja una tensión real, pero no puede justificar una incomprensión mutua. Un proyecto colectivo es también una multiplicidad de acciones de intervención individualizada, realizada con personas concretas, en situaciones específicas, y la evaluación debe reflejar esta realidad social para evitar tanto el espiritualismo ilusorio de las buenas intenciones como las medidas miopes que cuentan gestos pero no captan las transformaciones humanas, siempre más sutiles que cualquier indicador. La tarea no es fácil. Presencia física no siempre significa partipación, el que calla no necesariamente otorga...

Las dos experiencias de terreno, ambas de varios años de duración, tenían una intencionalidad abstracta común (la prevención del Sida), pero con formas muy distintas de acción. En un caso, las acciones se adaptaban a los contextos sociopolíticos de una región remota y económicamente desfavorecida de la región amazónica brasileña; en el otro, ellas se orientaban hacia medios urbanos y semiurbanos de un país social y económicamente favorecido con más recursos disponibles. En ambos, el trabajo fue concebido y realizado tratando de maximizar la participación en todas las etapas de las evaluaciones, desde la definición de metas y de medio, hasta la construcción de estrategias de recolección y de análisis de información y a la redacción de los informes. En ambas se generó un sector de mediación, de participantes que actuaron como agentes multiplicadores y de contacto.

En ambas fue posible romper la barrera de la palabra escrita, al menos reducir las desconfianzas hacia ella. Una medida de éxito de los proyectos es 
el grado en que la implicación de estos mediadores los hizo apropiarse de la experiencia, llevando su compromiso con ella mucho más allá que una simple obligación. Otra, igualmente provechosa, fue la generación de un discurso que los investigadores siempre sometieron a la consideración de esos colaboradores, que definieron su participación no sólo en las tareas a realizar sino también en la influencia que tenían en el producto final, que debía responder a sus criterios de claridad y de utilidad.

Dos desafíos se presentaron como cruciales. Por una parte, la definición implícita de la evaluación como investigación evocaba fantasmas de lenguaje abstruso, de estadísticas que provenían de indicadores impuestos. Por otra, los objetivos debían sobrepasar la idea de que eran necesariamente descendentes, que eran sólo la formulación de las exigencias externas de la financiación, para incorporar lo que era también la expresión de la dinámica de acción colectiva.

Toda reflexión sobre los métodos de evaluación está íntimamente ligada a opciones metodológicas que son necesariamente políticas (House, 2004). Ellas favorecen opciones específicas de acción colectiva que no pueden sino afectar la distribución del poder de producirlos y de difundirlos en una sociedad dada (Demo, 200o). Ellas quedan frecuentemente en la penumbra, tras bambalinas ideológicas, que permiten que las opciones políticas con las que se confunden puedan ser estigmatizadas como "extra científicas" - y por lo tanto puedan ser excluidas del análisis metodológico (Entwistle y col., 1998).

La contribución que la perspectiva de la sociedad civil popular puede hacer es importante y necesaria en la evaluación de la investigación en salud pública. Todavía, la "colonización de las posiciones de los "laicos" por la influencia bienhechora de los expertos ('expert speakers') y la posición híbrida del laico que llega a ser experto pueden ser características del proceso y del conservadurismo que emerge en esta tentativa de relación entre ambos. (Kerr y col., 2007). En un discurso alternativo que no ha sido nunca el dominante, la evaluación es más que el control administrativo de una modificación preconcebida, impuesta a los otros actores. También es necesario comprender cómo esta operación se inserta en las realidades locales del mundo cotidiano de los que son los actores responsables del éxito de la transformación buscada. Un objetivo predeterminado es un reflejo de lo que se sabe y se quiere tal como definido anticipadamente a partir de otros contextos, y también de lo que falta por saber para asegurar su eficacia en una situación en que se pretende encarnarlo, eficacia que sólo está asegurada si es el producto de una colaboración de todos los implicados.

La participación no es sólo un ideal abstracto: es también la comprensión de cómo un cambio afectará la vida de todos los que sean afectados por la propuesta de cambio (Demo, 2001). Este cambio no será sólo positivo, tampoco será aceptable por todos. Un proyecto de cambio social cambia mucho más de lo que quiere cambiar (Scriven, 2004), toca a muchos más que los que cree que toca y esta participación real de voluntades variadas determinará el efecto del proyecto mucho más que las cualidades intrínsecas de la idea que definía la intención de cambio de los conceptos y administradores del proyecto.

Es aquí donde la evaluación comunitaria es más que una invitación al compromiso político entre las concepciones globales de planificación de cambios sociales y la lógica de la participación igualitaria: es también un llamado a un realismo que comienza con el respeto de todos los que de hecho serán actores en el proyecto. Estos actores hablan de "valores" al enunciar los medios que favorecerán la eficacia de estos proyectos. Brandão (2006) describe la investigación participativa como un modo de aprender a escribir la historia colectiva a partir de una conciencia crítica de la propia.

Una tónica que ha marcado el análisis teórico de este trabajo ha sido la identificación de una tensión en las experiencias analizadas entre estructuras de encuadre de carácter globalista, universalista y gubernamental y la lucha por espacios de participación para los actores directamente implicados en la acción comunitaria. Ella enfrenta la lógica de programas genéricos (WHO, 1986, 2006; UNAIDS, 2005; UNAIDS/WB/UNDP, 2006; UNDP, 2004; ICASO, 2007) a la de las prácticas reales de intervención que buscan implementarlos, incorporando la información local necesaria para maximizar sus posibilidades de eficacia. Los modelos colonizadores buscan imponerse a situaciones que nunca fueron consideradas en su elaboración y confrontan la 
vitalidad real de acciones comunitarias, que pueden respetar las finalidades de los programas que realizan, pero que rehusan excluir de su análisis las condiciones concretas que limitan la aplicabilidad del modelo preconcebido. La ruptura posible entre las conceptualizaciones importadas y las acciones y los conceptos instrumentales en la acción directa ilustran dos lógicas de evaluación diferentes; si ellas son concebidas como opuestas e incompatibles, el costo para realizar un cambio real concreto es muy alto (Scriven, 2004).

\section{Perspectivas para las Ciencias de la Salud y Enfermeras}

La evaluación es una forma de conciencia práctica, herramienta indispensable en el arsenal del actor. En el caso de la evaluación comunitaria, la evaluación produce una voz, voz que es interna, de concertación de los esfuerzos de todos los participantes, y que es externa, destinada a ganar un espacio de visibilidad y de credibilidad para acciones que pueden estar oscurecidas por las voces que le son impuestas. La invitación a desarrollar una palabra es, sin embargo, engañosa. La palabra puede ser una afirmación, pero también puede ser utilizada para proteger lo amenazado: hay razones para hacerse oír y hay razones para callar. Opacidad y silencio son estrategias legítimas de ubicarse en un contexto social que puede ser hostil, represivo, peligroso. ¿Hacerse oír o callar?

La respuesta no es fácil - especialmente si no se confronta el dilema central de toda intervención, incluyendo la evaluable. ¿La palabra de quién? ¿A quién? ¿Para qué? El que evalúa - sea contralor, supervisor o facilitador - debe reconocer que su palabra moldea las palabras de los actores, sus experiencias. Si la evaluación es una palabra pública, también es una palabra firmada por un nuevo actor, participante influyente en la situación que reporta. Su contribución incluye sus competencias, y también sus intereses específicos. Silencio y opacidad - ¿Al servicio de quién - de los productores del discurso o de los sin voz?
Aún si el término puede parecer excesivo, un grupo comunitario puede tener un sentido profético, un don de visualizar un mundo diferente, más cercano a sus valores, y el que evalúa debe situarse existencialmente ante esta profecía. El silencio puede favorecer más fácilmente a quien evalúa, que al usuario, que vive los costos de su mutismo y de su invisibilidad. Y la investigación puede incorporar al discurso público los elementos que han quedado invisibles en las generalizaciones abstractas y en estadísticas agregadas.

Dar un espacio a la cotidianidad y a la particularidad en el discurso científico es asegurar que refleje más adecuadamente la realidad que pretende iluminar. La democratización de la producción y de la difusión de conocimientos crean hoy nuevas redes sociales por Internet (Skype, Twitter, Youtube, Facebook) que dan a una sociedad civil popular una voz en gestación que ayuda a hacer presiones para mejorar sus condicionnes de vida o para difundir sus prácticas innovadoras como puede verse en los ejemplos de congresos científicos internacionales de salud pública ${ }^{2}$ o en el campo de prácticas de formación profesional en salud realizadas en barrios populares (Cruz, 2007).

El artículo indica la importancia de la inclusión de la perspectiva de la acción social comunitaria de la sociedad civil popular en el desarrollo de acciones de salud pública por medio de las prácticas profesionales de los que actúan en forma coherente y comprometida junto con los grupos populares. Los profesionales de primera línea pueden comprometerse con los grupos comunitarios de la sociedad civil popular, como las asociaciones de mujeres, las trabajadoras del sexo, los agentes de pastoral sanitaria, etc. para incluirlos en la evaluación de la prevención sanitaria.

En este contexto, la práctica de la investigación científica puede incorporar la recuperación del saber popular y la conceptualización de las iniciativas alternativas en el "cuidar" y en el "saber cuidar". La inclusión en barrios periféricos y el trabajo con grupos comunitarios permite concretizar una investigación evaluable de las acciones sanitarias preventivas des-

2 Vasconcelos, M. Vídeo da participação do Movimento de Educação Popular e Saúde no Congresso Brasileiro de Ciências Sociais e Humanas na Saúde (Abrasquinho). Salvador, 2007. Disponível em: <http://br.youtube.com/watch?v=E5TFnNquPj8>. Acesso em: 18 jan. 2010. 
de una ética del compromiso social y de conciencia sociopolítica. La investigación científica llega a ser un servicio directo a la colectividad.

\section{Referencias}

ALBOAN. Guía 1. La sistematización, una nueva mirada a nuestras prácticas. Hegoa, 2006a. (Iniciativas de Cooperación y Desarrollo, Instituto de Derechos Humanos Padre Arrupe). Disponível em: <www.alboan.org/sistematizacion/?scc=6>. Acesso em: 27 aug. 2009.

ALBOAN. Guía 2. La aventura de la sistematización. Hegoa, 2006b (Iniciativas de Cooperación y Desarrollo, Instituto de Derechos Humanos Padre Arrupe). Disponível em: <www. alboan.org/sistematizacion/?scc=6 $>$. Acesso em: 27 aug. 2009.

ALVARADO, A.; MORENO, M. E.; RODRÍGUEZ, M. C. Inclusión social y participación comunitaria: una perspectiva de trabajo frente a la discapacidad. Ciencia e Enfermería, Concepcion, v. 15, n. 1, p. 61-74, 2009.

\section{ASÚN, D. La mediación comunitaria en} la intervención social. Sistematización y mediaciones: un análisis de experiencias de intervención en el campo de adicciones en Chile. In: CONGRESSO HABLEMOS DE DROGA, 2009, Barcelona, Anais... Barcelona, junio de 2009. Disponível em: <http://www. congresohablemosdedrogas.es/articulos2. php?products_id=70>. Acesso em: 15 jan. 2010.

BENZAKEN, A. S. et al. Baja prevalencia de DST en profesionales del sexo en un municipio del interior de Amazonas. Journal brasileiro de DST, Rio de Janeiro, v. 14, n. 4, p. 9-19, 2002.

BRANDÃO, C. R. A pesquisa participante e a participação da pesquisa: um olhar entre tempos e espaços a partir da América Latina. In: BRANDÃO, C. R; STRECK, D. R. (Orgs.). Pesquisa participante: o saber da partilha. Aparecida: Idéias \& Letras; 2006. p. 21-54.

CASANOVA, P. G. Exploração, colonialismo e luta pela democracia na América Latina. Petrópolis: Vozes, 2002.
CRUZ, P. J. Vídeo de divulgação do Projeto Educação Popular e Atenção à Saúde da Família - UFPB, na Comunidade Maria de Nazaré (João Pessoa - PB). Parte 2. João Pessoa, 2007. Disponível em: <http://www.youtube.com/ watch?v=VGvaF88WlWE $>$. Acceso em: 18 jan. 2010.

DEMO, P. Ambivalências da sociedade da informação. Ciência da Informação, Brasília, v. 29, n. 2, p.37-42, 2000.

DEMO, P. Participação é conquista. 5. ed. São Paulo: Cortez, 2001.

DEMO, P. Alfabetizações: desafios da nova mídia. Ensaio: avaliação e políticas públicas em Educação, Rio de Janeiro, v. 15, n. 57, p. 543-564, 2007.

DEMO, P. Pesquisa participante: saber pensar e intervir juntos. Brasilia: Liber Livro, 2008.

DIAMOND, J. Guns, germs, and steel. New York: WW Norton \& co., 1999.

EIZAGUIRRE, M.; URRUTIA, G.; ASKUNZE, G. La sistematización, nueva mirada a nuestras práticas: guía para la sistematización de experiencias de transformación social. Alboan: Hegoa e Instituto de Derechos Humanos Pedro Arrupe; Bilbao, 2004. Disponível em: <www. bantaba.ehu.es/lab/cont/doc/sistem1/>. Acesso em: 15 jan. 2010.

ENTWISTLE,V. A. et al. Lay perspectives: advantages for health research. BMJ: British medical journal, London, n. 316, n. 7129, p. 463466, 1998.

FARMER, P. Infections and inequalities: the modern plagues. Berkeley: The University of California Press, 2001.

FARMER, P. Pathologies of power: health, human Rights, and the new war on the poor. Berkley: University of California Press, 2003.

HOROWITZ, I. L. The rise and fall of Project Camelot: studies in the relationship between social science and practical politics. Cambridge: MIT Press, 1974. 
HOUSE, E. R. The role of the evaluator in a political word. The Canadian Journal of Programme Evaluation, Toronto, v. 19, n. 2, p. 1-16, 2004.

INTERNATIONAL COUNCIL OF AIDS SERVICES ORGANISATIONS [ICASO]. La coordinación con las comunidades: Parte A: Sobre la participación comunitaria: Directrices sobre la participación del Sector comunitario en la coordenacíon de las respuestas nacionales al SIDA. 2007. Disponível em: <http://www.icaso.org/index.html>. Acesso em: 15 jan. 2008.

JALBERT, Y. et al. Epsilon: a self-evaluation guide for community organizations. Montréal: COCQSida, 1997.

JARA HOLLAND, O. Sistematización de experiencias: caminos recorridos y nuevos horizontes. La Piragua - revista Latinoamericana de educación y de política, Ciudad de Panamá, n. 23, 2006. Disponível em: <http://ceaal.org/content/ view/234/114/>. Acceso em: 15 jan. 2010.

KERR, A.; CUNNINGHAM-BURLEY, S.; TUTTON, R. Shifting subject positions: experts and Lay people in public dialogue. Social Studies of Science, Thousand Oaks, v. 37, n. 3, p. 385-411, 2007.

LAPERRIÈRE, H. Evaluation of STD/HIV/ AIDS peer-education and dangerousness: a local perspective. Ciência e saúde coletiva, Rio de Janeiro, v. 13, n. 6, p. 1817-1824. 20o8a. Disponível em: <http://www.scielo. br/scielo.php?script=sci_arttext\&pid=S1413$81232008000600016 \& \operatorname{lng}=e n \& n r m=i s o>$. Acceso em: 2 set. 2011

LAPERRIÈRE, H. O caso de uma comunidade avaliativa emergente: reapropriação pelos pares-multiplicadores da avaliação das suas próprias ações preventivas e sociais contra as DST/HIV/AIDS. Interface - comunicação, saúde, educação, Botucatu, v. 12, n. 24, p.527540, 2008b. Disponível em: <http://www.scielo. br/scielo.php?script=sci_arttext\&pid=S1414$32832008000300006 \& \operatorname{lng}=e n \& n r m=i s o>$. Acceso em: 2 set. 2011.

PETTICREW, M. et al. Natural experiments: an underused tool for public health? Public Health, London, n. 119, n. 09, p. 751-757, 2005.
SALAS, A. S. Toward a North-South dialogue. Revisiting nursing theory (from the South). Advances in nursing sciences, Hagerstown, MD, v. 28, n. 1, p. 17-24, 2005.

SCRIVEN, M. Reflections. In: ALKIN, M. C. (Org.). Evaluation Roots: tracing Theorists' Views and Influences. Thousand Oaks: Sage, 2004, p.183-195.

SILVA, M. J.; BESSA, M. E. P. Conceitos de saúde e doença segundo a óptica dos idosos de baixa renda. Ciencia y enfermería, Concepción, v. 14, n. 1, p. 23-31, 2008.

STAVENHAGEN, R. Between underdevelopment and revolution: a Latin American perspective. New Delhi: Abhinav, 1981.

STAVENHAGEN, R. Cómo descolonizar las ciencias sociales. In: SALAZAR, M. C. (Org.). La investigación-ación participativa: inicios y desarrollos. Buenos Aires: Humanitas, 1992. p. 37-64.

TITTERTON, M.; SMART, H. Can participatory research be a route to empowerment? A case study of a disadvantaged Scottish community. Community development journal, Oxford, v. 43, n. 1, p. 52-64, 2008.

UNITED NATIONS DEVELOPMENT PROGRAMME [UNDP]. Decentralised governance for development: a combined practice note on decentralisation, local governance and urban/ rural development. Geneva, 2004. Disponível em: <www.undp.org/policy/docs/practicenotes/ dgdpnintra.doc $>$. Acesso em: 12 fev. 2007.

UNITED NATIONS JOINT PROGRAMME ON HIV AND AIDS [UNAIDS]. Mainstreaming HIV and AIDS in sectors \& programmes: an Implementation Guide for National responses. Geneva, 2005. Disponível em: <http://www.undp. org/hiv/hiv-humdev.htm>. Acesso em: 6 mar. 2006.

UNITED NATIONS JOINT PROGRAMME ON HIV AND AIDS/UNITED NATIONS DEVELOPMENT PROGRAMME/WORLD BANK [UNAIDS/UNDP/ WB]. Multisectorial \& local responses: moving from implementation to measuring results. Skillsbuilding session. International AIDS Conference, 16, Toronto, Canada, 2006. 
VALLA, V. V. A vida religiosa como estratégia das classes populares na América Latina de superação da situação de impasse que marca suas vidas. In: VASCONCELOS, E. M. (Org.). A espiritualidade no trabalho em saúde. São Paulo: Hucitec, 20o6. p. 265-295.

VASCONCELOS, E. M. Inserir, observar, intervir e refletir. Assim surge um programa de acompanhamento a familias em situação de risco. In: VASCONCELOS, E. M. Educação populare atenção à saúde da família. São Paulo: Hucitec, 2008. p. 79-175.

WORLD HEALTH ORGANISATIONS [WHO]. Ottawa Charter. Geneva, 1986. Disponível em: <http://www.idb.org/iuhpe/ottawa.htm>. Acesso em: 7 set. 2006.

WORLD HEALTH ORGANISATIONS [WHO]. The Bangkok charter for health promotion in a globalized world. Geneva, 2006. Disponível em: <http://www.who.int/healthpromotion/ conferences/6gchp/bangkok_charter/en/print. html>. Acesso em: 6 mar. 2006.
ZÚÑIGA, R. The experimenting society and radical social reform: the role of the social scientist in Chile's Unidad Popular experience. American Psychologist, Washington, DC, v. 30, n. 2, p. 99-115, 1976.

ZÚÑIGA, R. La subjetivación en la intervención comunitaria. In: JIMÉNEZ-DOMÍNGUES, B. (Org.). Subjetividad, participación e intervención comunitaria: una visión crítica desde América Latina. Buenos Aires: Paidós, 2008. p. 143-166.

ZÚÑIGA, R. Les recherches groupales dans l'action la systématisation d'expériences et le schéma SWOT en Amérique Latine. [Las investigaciones grupales en la acción: la sistematización de experiencias y el modelo FODA en América Latina]. Recherches qualitatives, Trois-Rivières (Québec), v. 28, n. 3, p. 112-134, 2009.

ZÚÑIGA, R.; LULY, M. H. Savoir-faire et savoir-dire: un guide d'évaluation communautaire. Montréal: COCQ-sida, 2005. 